\title{
Medical Comorbidities and Association With Mortality Risk in Alzheimer's Disease: Population-Based Study of 132,405 Geriatric Inpatients
}

Ting Yu Yen ${ }^{1}$, Nitya Beriwal ${ }^{2}$, Pawandeep Kaur ${ }^{3}$, Virendrasinh Ravat ${ }^{4}$, Rikinkumar S. Patel ${ }^{5}$

1. Medicine, Poznan University of Medical Sciences, Poznan, POL 2. Medicine, Lady Hardinge Medical College, New Delhi, IND 3. Medicine, Sri Guru Ram Das Institute of Medical Sciences and Research, Amritsar, IND 4. Internal Medicine, Krishna Institute of Medical Sciences, Karad, IND 5. Psychiatry, Griffin Memorial Hospital, Norman, USA

Corresponding author: Rikinkumar S. Patel, rpatel_09@arcadia.edu

\section{Abstract \\ Objectives}

We used the Nationwide Inpatient Sample (NIS) to identify the demographic predictors and study the impact of chronic comorbidities on the risk of in-hospital mortality in Alzheimer's disease (AD).

\section{Methods}

We included 132,405 AD patients from the NIS (2012-2014). We used descriptive statistics to discern the differences in demographics and comorbidities by in-hospital mortality. Logistic regression analysis was used to evaluate the predictors and impact of comorbidities that increase the risk of association with inhospital mortality.

\section{Results}

The in-hospital mortality in $\mathrm{AD}$ inpatients is $1.69 \%$, and a greater proportion were female (58.4\%) and white (81.5\%). Male and hispanic had a higher mortality risk than their counterparts. Hypertension (72\%) is the most prevalent comorbidity. Congestive cardiac failure (CCF) and renal failure were significantly associated with a higher risk of in-hospital mortality in $\mathrm{AD}$ inpatients by 1.4 and 1.5 times, respectively. Psychiatric comorbidities (depression $20.4 \%$, and psychosis $21.4 \%$ ) were prevalent in $\mathrm{AD}$ inpatients but were negatively associated with mortality. Comorbid tumors without metastasis (1.2\%) and metastatic cancer $(0.3 \%)$ were least prevalent but significantly increased the risk of in-hospital mortality by 1.6 times and 2.2 times, respectively.

\section{Conclusion}

Received 05/05/2020 Review began 05/13/2020 Review ended 05/13/2020 Published 05/19/2020

\section{() Copyright 2020}

Yen et al. This is an open access article distributed under the terms of the Creative Commons Attribution License CC-BY 4.0., which permits unrestricted use, distribution, and reproduction in any medium, provided the original author and source are credited.
CCF and renal failure were significantly associated with a higher risk of in-hospital mortality in AD patients. Less prevalent comorbidities, tumors with/without metastasis increased in-hospital mortality by $59 \%$ to $117 \%$. An integrated care model is required to manage comorbidities in AD patients to improve healthrelated quality of life and reduce morbidity and mortality.

Categories: Internal Medicine, Neurology, Preventive Medicine

Keywords: medical comorbidities, mortality, diastolic heart failure, congestive cardiac failure, alzheimer's disease, alzheimer's dementia, metastatic cancer, dementia

\section{Introduction}

Alzheimer's disease $(\mathrm{AD})$ is a progressive neurodegenerative disease that occurs due to the accumulation of abnormal protein folding inside the brain. $\mathrm{AD}$ accounts for $60 \%$ to $80 \%$ of the dementia cases [1]. Genetic mutation is mainly involved in early-onset $\mathrm{AD}$ patients, and the combination of genetic, environmental, and lifestyle factors are linked to $\mathrm{AD}$ [2]. Aging has been known as the most important risk factor, as the number of patients with $\mathrm{AD}$ doubles every five years after the age of 65 , and above 85 consists of about one-third of the $\mathrm{AD}$ population [2]. In 2020, it is estimated that more than 5.5 million Americans suffer from $\mathrm{AD}$ and it is expected to increase to 14 million by 2060 [3]. An estimated annual cost per AD patient is $\$ 41,000$ to $\$ 56,000$, with a total cost of $\$ 159$ billion to $\$ 215$ billion nationwide in 2010 [4].

Among the $\mathrm{AD}$ patients, beta-amyloid plaques and the neurofibrillary tangles in the brain are more widespread and predominant than the elderly patients without $\mathrm{AD}$ [5]. These changes in the brain impair the normal neuronal functioning and ultimately lead to disconnections with other neurons [2]. AD is the sixth leading cause of death in the United States (US), and the fifth cause for those aged above 65 years [6]. In 2018, there were 122,019 deaths among those suffering from AD, according to the Centers for Disease 
Control and Prevention (CDC). However, the number of death from dementia was much higher, about 266,957 in total [7].

Bronchopneumonia is the most common cause of death in a patient with AD due to impaired swallowing ability, inability to clear airways secretions, apathies, and decreased immune function may all be crucial as the underlying causes for bronchopneumonia [8]. This is followed by circulatory system disease with ischemic heart disease accounting for more than $60 \%$ of deaths and cancer. Lack of oxygen supply in pneumonia patients with $\mathrm{AD}$ may contribute to non-symptomatic coronary atherosclerosis resulting in higher inpatient mortality [8]. Several studies have demonstrated an inverse relationship between neurodegenerative disease and cancer; however, underlying pathology behind this remains unknown [9].

We used the US national hospital data to identify the demographic predictors of in-hospital mortality in AD inpatients and the impact of chronic comorbidities on the risk of in-hospital mortality.

\section{Materials And Methods}

\section{Data source}

Cross-sectional data analysis was done using the Nationwide Inpatient Sample (NIS, 2012 to 2014) [10]. This dataset includes clinical information during the hospitalization from 4,400 non-federal hospitals and covers about 44 states in the US [10]. Diagnostic information is coded using the International Classification of Diseases, ninth edition (ICD-9) diagnostic codes, and Clinical Classification Software (CCS) codes. The NIS is a publicly available de-identified database and so doesn't require approval from the institutional review board [10].

\section{Inclusion criteria and outcome variables}

We included 132,405 inpatients with a primary discharge diagnosis of AD using ICD-9 code 331.0. Demographic variables included were age, sex, and race (white, black, hispanic, and others) [11]. The chronic comorbidities including diabetes, hypertension, congestive cardiac failure (CCF), chronic pulmonary disease, renal failure, depression, psychosis, and tumor with and without metastasis were identified using ICD-9 or CCS diagnosis codes [11]. The in-hospital mortality in the study population is all-cause [11].

\section{Statistical analysis}

We used descriptive statistics to discern the differences in demographics and comorbidities by in-hospital mortality. Logistic regression analysis was used to evaluate the demographic predictors and impact of comorbidities that increases the risk of association with in-hospital mortality. A P-value of less than 0.01 was considered statistical significance in our data analysis models that were done in the Statistical Package for the Social Sciences (SPSS), version 26 (IBM Corporation, Armonk, NY).

\section{Results}

We analyzed a total sample of 132,405 AD inpatients with an in-hospital mortality of $1.69 \%(\mathrm{~N}=2,245)$. The majority of the $\mathrm{AD}$ inpatients were female (58.4\%) and white (81.5\%). The most prevalent chronic comorbidities were hypertension (72\%), as shown in Figure 1.

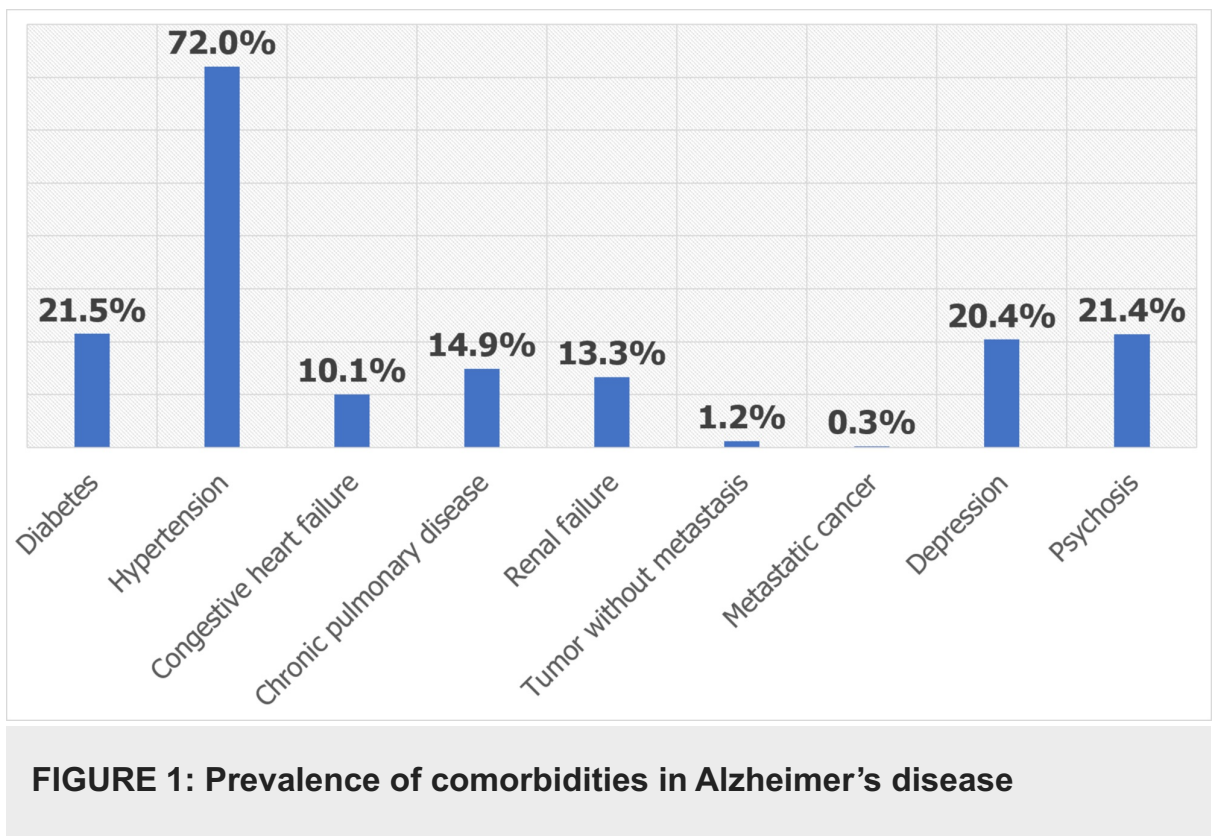




\section{Cureus}

\section{inpatients}

\section{Risk factors for in-hospital mortality}

The mean age of AD inpatients who died during hospitalization were older ( 83.5 vs. 80.6 years). Males had 1.2 times higher odds for deaths (95\% CI 1.069-1.276) than females. Though $78.2 \%$ of Whites died during hospitalization, in the regression model, hispanics had two times (95\% CI 1.617-2.210) higher risk of mortality compared to whites.

CCF and renal failure were significantly associated with a higher risk of in-hospital mortality in AD inpatients by 1.4 to 1.5 times. Psychiatric comorbidities (depression $20.4 \%$, and psychosis $21.4 \%$ ) were prevalent in $\mathrm{AD}$ inpatients but were negatively associated with mortality. Comorbid tumor without metastasis $(1.2 \%)$ and metastatic cancer $(0.3 \%)$ were least prevalent but significantly increases the risk of inhospital mortality by 1.6 times (95\% CI 1.193-2.128) and 2.2 times (95\% CI $1.285-3.653$ ), respectively, after controlling for demographic and other comorbidities as shown in Table 1.

\begin{tabular}{|c|c|c|c|c|c|}
\hline \multirow{2}{*}{ Variable } & \multicolumn{2}{|c|}{ In-hospital mortality, \% } & \multicolumn{3}{|c|}{ Logistic regression model } \\
\hline & No & Yes & OR & $95 \% \mathrm{Cl}$ & P-value \\
\hline Total (N) & 130,155 & 2,245 & - & - & - \\
\hline Mean age, years (SD) & $80.6(7.72)$ & $83.5(6.19)$ & 1.06 & $1.055-1.070$ & $<0.001$ \\
\hline \multicolumn{6}{|l|}{ Sex } \\
\hline Male & 41.6 & 45.9 & 1.17 & $1.069-1.276$ & $<0.001$ \\
\hline Female & 58.4 & 54.1 & \multicolumn{3}{|c|}{ Reference } \\
\hline \multicolumn{6}{|l|}{ Race } \\
\hline White & 81.5 & 78.2 & \multicolumn{3}{|c|}{ Reference } \\
\hline Black & 9.7 & 7.7 & 0.96 & $0.811-1.130$ & 0.609 \\
\hline Hispanic & 5.1 & 8.9 & 1.89 & $1.617-2.210$ & $<0.001$ \\
\hline Other & 3.8 & 5.3 & 1.49 & $1.228-1.822$ & $<0.001$ \\
\hline \multicolumn{6}{|l|}{ Comorbidities } \\
\hline No comorbidities & - & - & \multicolumn{3}{|c|}{ Reference } \\
\hline Diabetes & 21.5 & 19.8 & 1.13 & $1.013-1.260$ & 0.029 \\
\hline Hypertension & 72.3 & 53.0 & 0.39 & $0.356-0.427$ & $<0.001$ \\
\hline Congestive cardiac failure & 10.0 & 13.6 & 1.41 & $1.237-1.599$ & $<0.001$ \\
\hline Chronic pulmonary disease & 15.0 & 11.8 & 0.84 & $0.733-0.956$ & 0.009 \\
\hline Renal failure & 13.3 & 17.6 & 1.48 & $1.310-1.661$ & $<0.001$ \\
\hline Tumor without metastasis & 1.2 & 2.2 & 1.59 & $1.193-2.128$ & 0.002 \\
\hline Metastatic cancer & 0.3 & 0.7 & 2.17 & $1.285-3.653$ & 0.004 \\
\hline Depression & 20.5 & 14.7 & 0.68 & $0.595-0.766$ & $<0.001$ \\
\hline Psychosis & 21.6 & 10.0 & 0.47 & $0.404-0.539$ & $<0.001$ \\
\hline
\end{tabular}

TABLE 1: Risk factors of in-hospital mortality in Alzheimer's disease inpatients

$\mathrm{N}$ : number of patients; OR: odds ratio

\section{Discussion}

In our inpatient study, the mean age of $\mathrm{AD}$ inpatients who died during hospitalization was 83.5 years. As per 
a study, $\mathrm{AD}$ is prevalent in the age above 90 years and is projected to increase rapidly [12]. Male $\mathrm{AD}$ patients had a $17 \%$ higher risk of mortality, which is supported by the Framingham heart study; however, the causal relationship remains speculative [13]. Our AD inpatients from the hispanic race group had higher in-hospital mortality increased by $89 \%$. As per the National Alzheimer's Coordinating Center data from 30 AD centers in the US $(\mathrm{N}=30,916)$ found that Latino and blacks with AD had a longer survival time compared to the whites [14]. Yet, this study had some limitations, including a lack of uniform process for data acquisition and selection bias [14]. Whites have better access to healthcare for the management of comorbidities and have a higher rate of nursing home placement due to which in our study we found that whites had a lower risk of mortality compared to minority groups [15].

Hypertension is the most prevalent chronic comorbidities (72\%), followed by diabetes (21.5\%), psychosis (21.4\%), and depression in our study $\mathrm{AD}$ inpatients. In a study comparing the comorbidities of young-onset versus late-onset $\mathrm{AD}$, circulatory disease and hypertension were most prevalent in both [16]. This is followed by psychiatric disorders, and endocrine and metabolic disorders, and diabetes [16]. Notably, the late-onset $\mathrm{AD}$ patients had a significantly higher prevalence rate of hypertension (61.2\% vs. 35\% in early-onset) and diabetes (20.9\% vs. $7.8 \%$ in early-onset) [16]. Aging increases the prevalence rate of these chronic comorbidities, which are also risk factors for AD's progression [17]. Lung diseases, other forms of heart disease, and disease of the genitourinary system are remarkable comorbidity in AD patients; however, they are less prevalent as also seen in our study [16].

The macrovascular and microvascular damage from hypertension and diabetes, consequently, lead to CCF and renal failure. Glomerular hyperfiltration, overactive renin-angiotensin-aldosterone system, and renal dynamic changes may all contribute to renal disease [18]. In our study, we did not find a significant association between hypertension and diabetes and in-hospital mortality, but renal failure and CCF significantly increases mortality risk by $48 \%$ and $41 \%$, respectively. Some studies in Parkinson's disease found that depression and psychosis worsen hospitalization outcomes and disease severity, but this was not seen in our $\mathrm{AD}$ patients and was not associated with increased mortality risk [19,20].

Multiple studies suggested that AD is inversely associated with cancer-specific mortality [9,21]. The pairedhelical-filament (PHF)-tau tangles were lower in AD patients with cancer, and PHF-tau tangles correlate to cognitive impairment and dementia severity [21]. Cancer patients may have enhanced immune function to reliant $\mathrm{AD}$ progression, better lifestyle choices, and possible PHF-tau protein reduction from chemotherapy [21]. However, our study has demonstrated that metastatic and non-metastatic tumor increases the risk of in-hospital mortality by $117 \%$ and $59 \%$, respectively, after controlling for demographic confounders and chronic comorbidities.

There are some limitations to our study. Our inpatient population was predominantly white and so may lack population diversity and may not represent the general population. Second, the healthcare availability to minority populations may be underreported in our study sample. Lastly, the inconsistency and reporting bias about using the ICD-9 codes in the database, and lack of causal relationship between mortality in AD and comorbidities. Regardless of this, we have used a large sample population from the hospitals across 44 states in the US with national representation and found an epidemiological association between chronic comorbidities worsening mortality risk in $\mathrm{AD}$ patients.

\section{Conclusions}

About four-fifth of $\mathrm{AD}$ inpatients were white and prevalent comorbidities included hypertension and diabetes. CCF and renal failure were significantly associated with a higher risk of in-hospital mortality in AD patients by $41 \%$ and $48 \%$, respectively. In addition, the less prevalent comorbid tumor with/without metastasis also increased the in-hospital mortality by $59 \%$ to $117 \%$. Psychiatric comorbidities were prevalent (20\% to $21 \%$ ) but were negatively associated with mortality risk. An integrated care model is required to manage medical and psychiatric comorbidities in $\mathrm{AD}$ patients to improve health-related quality of life and reduce morbidity and mortality.

\section{Additional Information \\ Disclosures}

Human subjects: Consent was obtained by all participants in this study. Animal subjects: All authors have confirmed that this study did not involve animal subjects or tissue. Conflicts of interest: In compliance with the ICMJE uniform disclosure form, all authors declare the following: Payment/services info: All authors have declared that no financial support was received from any organization for the submitted work. Financial relationships: All authors have declared that they have no financial relationships at present or within the previous three years with any organizations that might have an interest in the submitted work. Other relationships: All authors have declared that there are no other relationships or activities that could appear to have influenced the submitted work.

\section{References}

1. Alzheimer's and dementia: facts and figures . (2020). Accessed: April 10, 2020: 
https://www.alz.org/alzheimers-dementia/facts-figures.

2. What causes alzheimer's disease?. (2019). Accessed: April 10, 2020: https://www.nia.nih.gov/health/whatcauses-alzheimers-disease.

3. Alzheimer's disease fact sheet. (2019). Accessed: April 10, 2020: https://www.nia.nih.gov/health/alzheimers-disease-fact-sheet.

4. Hurd MD, Martorell P, Delavande A, Mullen KJ, Langa KM: Monetary costs of dementia in the United States . N Engl J Med. 2013, 368:1326-1334. 10.1056/NEJMsa1204629

5. Causes of dementia. (2019). Accessed: April 10, 2020: https://www.dementia.org.au/aboutdementia/dementia-research/causes-of-dementia.

6. Leading causes of death and numbers of deaths, by age: United States, 1980 and 2017 . (2018). Accessed: April 10, 2020: https://www.cdc.gov/nchs/data/hus/2018/007.pdf.

7. National vital statistics reports. (2019). Accessed: April 10, 2020: https://www.cdc.gov/nchs/data/nvsr/nvsr68/nvsr68_02-508.pdf.

8. Heun R, Schoepf D, Potluri R, Natalwala A: Alzheimer's disease and co-morbidity: increased prevalence and possible risk factors of excess mortality in a naturalistic 7-year follow-up. Eur Psychiatry. 2013, 28:40-48. 10.1016/j.eurpsy.2011.06.001

9. Romero JP, Benito-Leon J, Louis ED, Bermejo-Pareja F: Alzheimer's disease is associated with decreased risk of cancer-specific mortality: a prospective study (NEDICES). J Alzheimers Dis. 2014, 40:465-473. 10.3233/JAD-132048

10. Overview of the national (nationwide) inpatient sample . (2019). Accessed: March 15, 2020: https://www.hcup-us.ahrq.gov/nisoverview.jsp.

11. NIS description of data elements . (2019). Accessed: March 15, 2020: https://www.hcupus.ahrq.gov/db/nation/nis/nisdde.jsp.

12. Kato K, Shirosita K, Kurosawa S, et al.: Staphylococcal enterotoxin a induced interferon (ifn)-gamma production in spleen cells from bcg-immunized mice: the ifn production is dependent on leukotriene c4 but not dependent on interleukin 2. Immunobiology. 1990, 181:40-50. 10.1016/s0171-2985(11)80164-6

13. Chene G, Beiser A, Au R, Preis SR, Wolf PA, Dufouil C, Seshadri S: Gender and incidence of dementia in the Framingham Heart Study from mid-adult life. Alzheimer's Dement. 2015, 11:310-320. 10.1016/j.jalz.2013.10.005

14. Mehta KM, Yaffe K, Perez-Stable EJ, Stewart A, Barnes D, Kurland BF, Miller BL: Race/ethnic differences in ad survival in US Alzheimer's disease centers. Neurology. 2008, 70:1163-1170. 10.1212/01.wnl.0000285287.99923.3c

15. Stern Y, Tang MX, Albert MS, et al.: Predicting time to nursing home care and death in individuals with Alzheimer disease. JAMA. 1997, 277:806-812. 10.1001/jama.1997.03540340040030

16. Gerritsen AA, Bakker C, Verhey FR, de Vugt ME, Melis RJ, Koopmans RT, 4C study team: Prevalence of comorbidity in patients with young-onset alzheimer disease compared with late-onset: a comparative cohort study. J Am Med Dir Assoc. 2016, 17:318-323. 10.1016/j.jamda.2015.11.011

17. Norton S, Matthews FE, Barnes DE, Yaffe K, Brayne C: Potential for primary prevention of alzheimer's disease: an analysis of population-based data. Lancet Neurol. 2014, 13:788-794. 10.1016/S14744422(14)70136-X

18. Lin YC, Chang YH, Yang SY, Wu KD, Chu TS: Update of pathophysiology and management of diabetic kidney disease. J Formos Med Assoc. 2018, 117:662-675. 10.1016/j.jfma.2018.02.007

19. Imran S, Patel RS, Onyeaka HK, et al.: Comorbid depression and psychosis in Parkinson's disease: a report of 62,783 hospitalizations in the United States. Cureus. 2019, 11:e5227. 10.7759/cureus.5227

20. Patel RS, Makani R, Mansuri Z, Patel U, Desai R, Chopra A: Impact of depression on hospitalization and related outcomes for Parkinson's disease patients: a nationwide inpatient sample-based retrospective study. Cureus. 2017, 9:e1648. 10.7759/cureus.1648

21. Yarchoan M, James BD, Shah RC, et al.: Association of cancer history with Alzheimer's disease dementia and neuropathology. J Alzheimers Dis. 2017, 56:699-706. 10.3233/JAD-160977 